\title{
The cyanobacterial endosymbiont of the unicellular algae Rhopalodia gibba shows reductive genome evolution Christoph Kneip ${ }^{\dagger 1,2}$, Christine Vo $\beta^{\dagger 1}$, Peter J Lockhart ${ }^{3}$ and Uwe G Maier*1
}

Address: ${ }^{1}$ Department of Cell Biology, Philipps-University Marburg, Marburg, Germany, ${ }^{2}$ Present address: Department of Molecular Biology, MaxPlanck-Institute for Infection Biology, Berlin, Germany and ${ }^{3}$ Allan Wilson Centre for Molecular Ecology and Evolution, Institute of Molecular BioSciences, Massey University, Palmerston North, New Zealand

Email: Christoph Kneip - kneip@mpiib-berlin.mpg.de; Christine Voß - Christine.Voss@staff.uni-marburg.de; Peter J Lockhart - p.j.lockhart@massey.ac.nz; Uwe G Maier* - maier@staff.uni-marburg.de

* Corresponding author †Equal contributors

Published: 28 January 2008

BMC Evolutionary Biology 2008, 8:30 doi:10.1 I86/147|-2148-8-30

This article is available from: http://www.biomedcentral.com/I47I-2/48/8/30

(c) 2008 Kneip et al; licensee BioMed Central Ltd.

This is an Open Access article distributed under the terms of the Creative Commons Attribution License (http://creativecommons.org/licenses/by/2.0), which permits unrestricted use, distribution, and reproduction in any medium, provided the original work is properly cited.
Received: 6 June 2007

Accepted: 28 January 2008

\begin{abstract}
Background: Bacteria occur in facultative association and intracellular symbiosis with a diversity of eukaryotic hosts. Recently, we have helped to characterise an intracellular nitrogen fixing bacterium, the so-called spheroid body, located within the diatom Rhopalodia gibba. Spheroid bodies are of cyanobacterial origin and exhibit features that suggest physiological adaptation to their intracellular life style. To investigate the genome modifications that have accompanied the process of endosymbiosis, here we compare gene structure, content and organisation in spheroid body and cyanobacterial genomes.
\end{abstract}

Results: Comparison of the spheroid body's genome sequence with corresponding regions of near free-living relatives indicates that multiple modifications have occurred in the endosymbiont's genome. These include localised changes that have led to elimination of some genes. This gene loss has been accompanied either by deletion of the respective DNA region or replacement with noncoding DNA that is AT rich in composition. In addition, genome modifications have led to the fusion and truncation of genes. We also report that in the spheroid body's genome there is an accumulation of deleterious mutations in genes for cell wall biosynthesis and processes controlled by transposases. Interestingly, the formation of pseudogenes in the spheroid body has occurred in the presence of intact, and presumably functional, recA and recF genes. This is in contrast to the situation in most investigated obligate intracellular bacterium-eukaryote symbioses, where at least either recA or recF has been eliminated.

Conclusion: Our analyses suggest highly specific targeting/loss of individual genes during the process of genome reduction and establishment of a cyanobacterial endosymbiont inside a eukaryotic cell. Our findings confirm, at the genome level, earlier speculation on the obligate intracellular status of the spheroid body in Rhopalodia gibba. This association is the first example of an obligate cyanobacterial symbiosis involving nitrogen fixation for which genomic data are available. It represents a new model system to study molecular adaptations of genome evolution that accompany a switch from free-living to intracellular existence. 


\section{Background}

A diversity of extracellular and intracellular symbiotic interactions occurs between bacteria and eukaryote hosts. The degree of interconnection between partners ranges from the weak dependence of some extracellular associations to permanent or obligate intracellular symbiosis. In the latter case, the endosymbiont is transmitted vertically to the next generation without any need for re-infection. The dependence on the host can be stabilised by loss or inactivation of genes, whose products are no longer required in the partnership $[1,2]$. Consequently, intracellular obligate symbionts loose their autonomy and therefore the capacity for a host-independent life style. Isolated from free-living populations, vertically transmitted endosymbionts have limited possibilities for genetic exchange through processes such as conjugation or transformation. Typically, endosymbiont genes diverge rapidly in comparison to their homologues in free-living relatives, a phenomenon that perhaps reflects genetic drift operating on small population size $[3,4]$ and/or relaxation of structural/functional constraints on endosymbiont protein evolution [5]. The genomes of obligate intracellular bacteria often show an accumulation of deleterious mutations and a higher AT-ratio, accompanied with reduction in genome size when compared to their free-living relatives [6]. The dimension of these processes can be as extreme as seen in the reduced genome of Buchnera sp., an endosymbiont of aphids with a genome size of $641 \mathrm{kbp}[7,8]$ and Carsonella, a $\gamma$-proteobacterial symbiont of phloem sapfeeding insects with a genome size of only $160 \mathrm{kbp}$ [9]. Others, like the endosymbionts of the rice weevils Sitophilus zeamais (SZPE) and Sitophilus oryzae (SOPE) as well as Sodalis glossinidius, a symbiont of tsetse flies [1012] represent the other extreme, and exhibit only slight reduction in genome size in comparison to free-living close relatives. Unlike the genomes of Buchnera and Carsonella, the genomes of these endosymbionts do not exhibit unusually high AT content.

Intracellular symbionts including SZPE and SOPE, have lost at least one of the recombinational repair enzyme genes encoded by recA and $r e c \mathrm{~F}$, a characteristic found in all other bacterial intracellular symbionts. The only known exception to this finding is the observation of intact genes for $r e c \mathrm{~A}$ and $r e c \mathrm{~F}$ in $\mathrm{S}$. glossinidius. The occurrence of genes for flagella apparatus still encoded in the genome of $S$. glossinidus might indicate that this symbiosis has only recently been established [12]. Cyanobacterial interactions with plants and protists are also well known [13-17]. These associations are in most cases facultative, and do not involve vertical transmission. In these cases, the cyanobacterial symbiont re-infects the host every generation. As with other facultative symbioses, genetic modification of the endosymbiont genome is yet undetected [17].
The pennate diatom Rhopalodia gibba harbours endosymbionts closely related to extant cyanobacteria. Some of the closest free-living relatives of these so-called spheroid bodies are diazotrophic cyanobacteria of the Cyanothece sp. group [18]. The spheroid bodies encode genes for nitrogen fixation and have the capacity to fix molecular nitrogen $[18,19]$. Although the spheroid bodies are of cyanobacterial origin, they lack the typical photosynthetic pigmentation; and thus have been assumed to be photosynthetically inactive. Unlike all other unicellular nitrogen fixing cyanobacteria, they fix nitrogen under light conditions only [18-20]. We observe one to four spheroid bodies per host cell depending on culture conditions which are transmitted vertically to the daughter cells during host cell division [15]. Altogether, these findings have led to the supposition that the spheroid bodies of $R$. gibba are obligate endosymbionts. Physiological adaptation to an intracellular endosymbiotic association is expected to result in genome modification and this expectation has motivated our investigation of gene structure, content and organisation in the spheroid body's genome of $R$. gibba. In order to investigate this, we have constructed fosmid libraries of the spheroid body and Cyanothece sp. ATCC 51142 and analysed genomic regions of special interest. Here we describe observations and analyses of the nifgene region and also loci relevant to the question of the obligate nature of the spheroid body endosymbiosis. Our investigations show massive genomic changes introduced into the spheroid body's genome. These include inactivation and losses of genes and the creation of large non-coding AT-rich areas. Our observations confirm the obligate nature of the spheroid body endosymbiont and provide insight into the nature of genome changes that accompany endosymbiosis and organelle formation.

\section{Results \\ The nif-gene region of spheroid bodies and Cyanothece sp. ATCC 5 II 142}

For this study we cloned and sequenced the nif-operon and flanking regions from the genomes of the intracellular symbiont of the diatom $R$. gibba, the spheroid bodies, and a close free-living relative, the diazotrophic cyanobacterium Cyanothece sp. ATCC 51142 (Figure 1). Altogether we sequenced and analysed a contiguous 63,362 bp Cyanothece fragment comprising the nif gene region and a contiguous 51,475 bp fragment including the corresponding region of the spheroid body's genome. Additionally, we sequenced and analysed 140,000 bp of non-contiguous genomic DNA of the endosymbiont. Using these additional datasets we characterised sequences in the spheroid body's genome for $r e c \mathrm{~A}, r e c \mathrm{~F}, p s b \mathrm{C}$ and $p s b \mathrm{D}$. For further analyses we also used information from the genome of the recently sequenced Cyanothece strain CCY0110 and other closely related cyanobacteria whose genomes have been sequenced and are available in the NCBI nr data- 


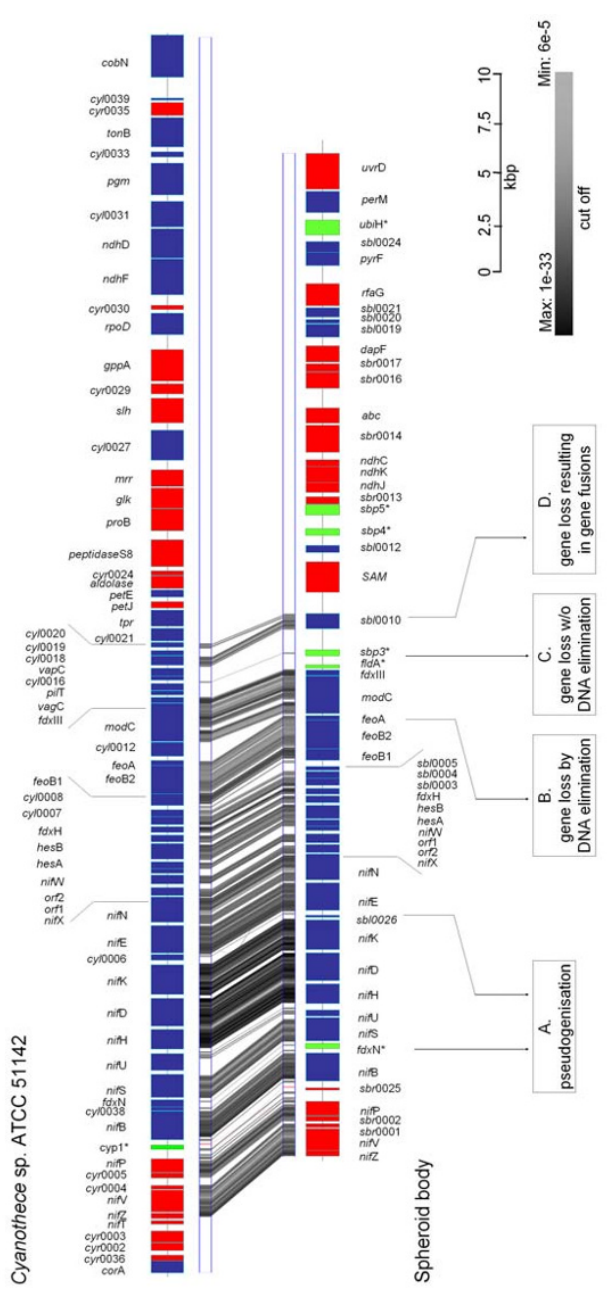

Figure I

Gene content in, and downstream of, the nif gene region of Cyanothece sp. ATCC 5 I I42 and spheroid body of $\boldsymbol{R}$. gibba. Blue and red bars represent orfs coded on the leading or lagging strand of DNA, respectively. The locations of pseudogenes in the spheroid body fragment have been indicated with green bars. Genes have been named either according to homology matches in BLAST analyses or numbered consecutively for each organism (see also additional files I and 2). A GATA [29] plot is shown and indicates regions of high synteny between both organisms. GATaligner settings were: Window size: 100; Match: 5; MisMatch:-4; Gap Creation:- 10; Gap Extension:-4; Raw Score Cut Off: 80. GATAPlotter score settings: Max: I4I bits, expect IE-33; Min: 46.8 bits, expect 5E-5. GATAPlotter scores have been represented using a greyscale bar. Regions of the spheroid body genome showing modifications of special interest have been indicated. A) Gene inactivation by pseudogenisation (e.g. $\left.f d x N^{*}\right)$; B) Gene deletion with DNA loss (e.g. cyl00I2); C) Gene deletion without DNA loss resulting in large noncoding regions (e.g. cyl00 I6); D) Gene deletion with DNA loss resulting in gene fusion (e.g. cy/0019). See text for further description of individual modifications. base. To obtain a phylogenetic framework for making inference of genome modification in the spheroid body's genome we reconstructed maximum likelihood gene trees for all homologues greater than 200 amino acids in the 63,362 bp region. Where possible, trees were outgroup rooted using homologues from Synechocystis sp. PCC 6803. Figure 2 shows supernetworks [21] built for these taxa. These networks summarise the relationships in individual gene trees which do not necessarily need to have the same taxon sampling. In this analysis, with some proteins (NifB, NifN, NifS), the spheroid body's genome was found to be most closely related to Cyanothece sp. ATCC 8801 (Figure 2a) but with other proteins (NifD, NifH, NifK, NifE), spheroid body sequences have a closer phylogenetic relationship with Cyanothece sp ATCC 51142, Cyanothece strain CCY0110, Crocosphaera watsonii WH 8501, and Gloeothece sp. KO68DGA (Figure 2b).

Figure 1 summarises the nif-operon-related regions of Cyanothece sp ATCC 51142 and the spheroid body's genome. In both, components of the nitrogenase dependent cyanobacterial nitrogen fixation machinery are encoded, including structural genes of the nitrogenase $n i f \mathrm{H}, n i f \mathrm{D}$ and $n i f \mathrm{~K}$, cofactors $(n i f \mathrm{~B}, n i f \mathrm{~N}, n i f \mathrm{E}, n i f \mathrm{~V}, n i f \mathrm{~W})$ and processing proteins for metal centre biosynthesis (nifU, nifS). As shown in figure 1, synteny of the nif-genes including the size of intergenic regions is very high. There is an overall $\mathrm{G} / \mathrm{C}$-content of $40.8 \%$ for this region of Cyanothece genome and a G/C-content of $37.2 \%$ for the spheroid body sequence. Codon usage is nearly equivalent in both genome regions with a slight AT-bias at the third codon position in the spheroid body genes (see Table 1).

Although the spheroid body's genome contains the same set of genes at the nif locus as in Cyanothece ATCC 51142, remarkable differences are apparent. Notably, between $n i f \mathrm{~B}$ and nifS, the functional $f d x \mathrm{~N}$ gene is replaced by a pseudogene $\left(f d x \mathrm{~N}^{*}\right)$ in the spheroid body's genome. The coding sequence is interrupted by several stop-codons. In contrast, an intact reading frame for $f d x \mathrm{~N}$ is conserved in all other close free-living cyanobacterial relatives, indicating formation of the pseudogene is a derived feature of the spheroid body lineage (Figure 3 ). The presence of a truncated nifU gene is also derived on the endosymbiont lineage, corresponding to approximately 170 amino acids of the N-terminus (Figure 4). This nif U homologue still encodes an intact open reading frame for the [2Fe-2S] binding and C-terminal NifU-domain. Interesting, in more distantly related cyanobacteria (Synechocystis sp. PCC 6803 and Gloeobacter violaceus PCC 7421) a truncated homologue is also present. Based on the phylogenetic analyses reported in Figure 2 and also comparative analyses of the NifU protein (not shown) it appears that truncation of nifU in spheroid bodies is a derived feature of the endosymbiont lineage. 


\section{A.}

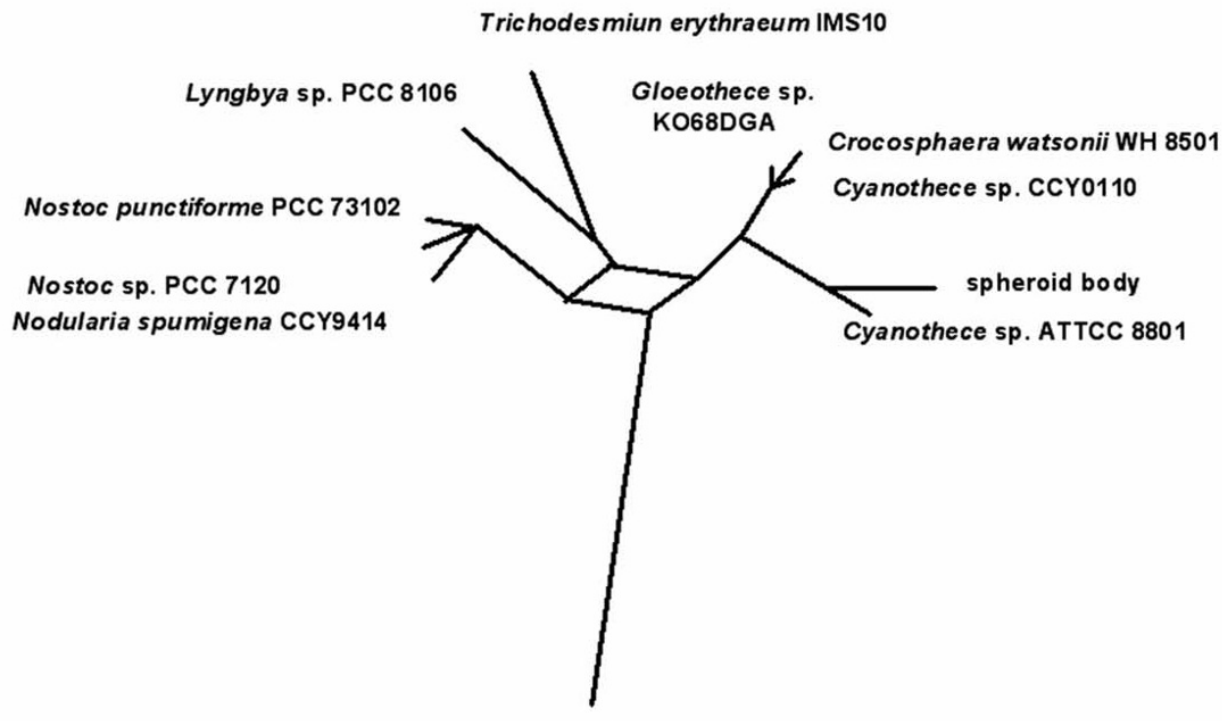

Synechocystis PCC 6803

B.

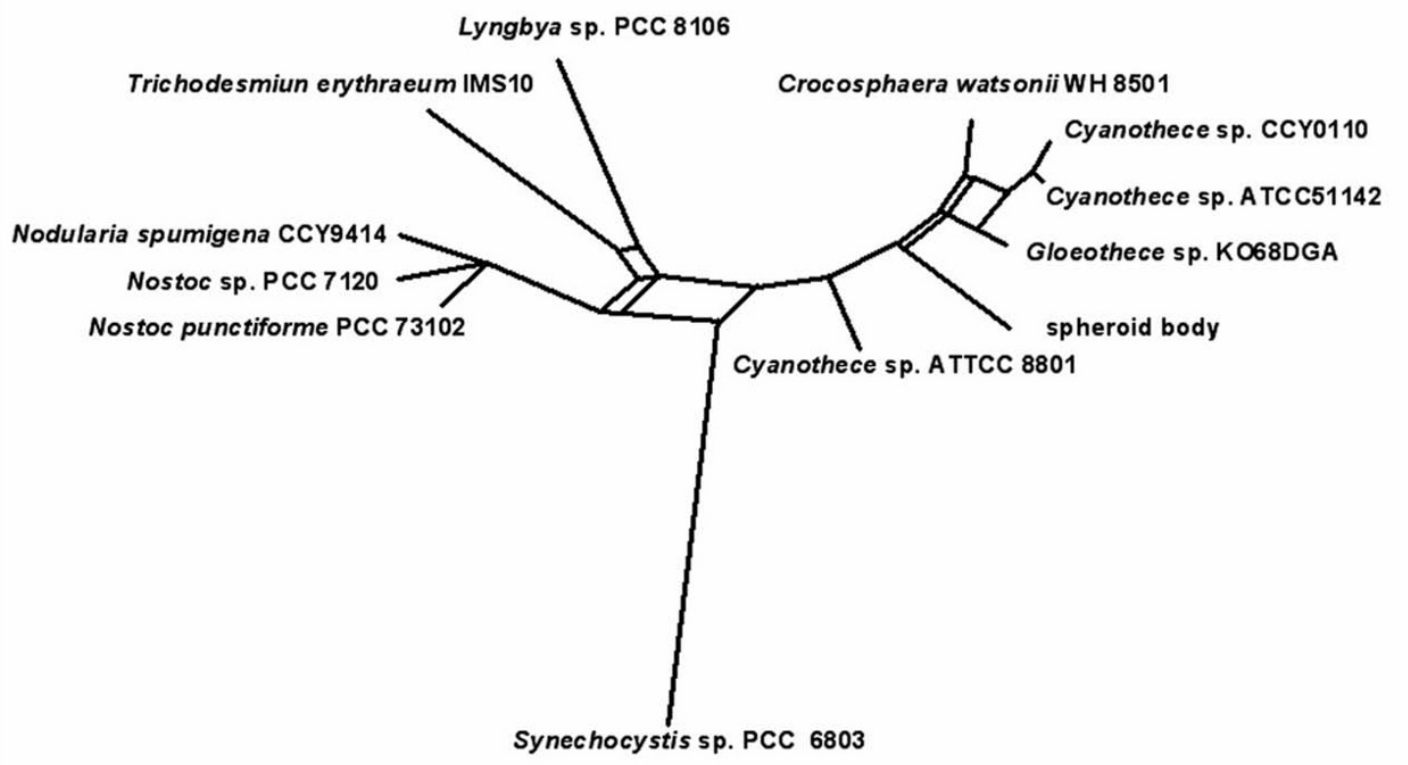

Figure 2

Supernetworks displaying relationships inferred from phylogenetic analysis of different gene regions. A. Supernetwork reconstructed using strict consensus maximum likelihood trees for NifB, NifN, NifS and ABC, HesA, NdhK, ModC, PerM, UvrD, SAM, Sbr00I4 of different cyanobacetria (see Materials and Methods). B. Supernetwork reconstructed using strict consensus maximum likelihood trees for NifD, NifH, NifK, NifE, and ABC, HesA, NdhK, ModC, PerM, UvrD, SAM, Sbr00I4. The networks are outgroup rooted using Synechocystis PCC 6803. A reticulation occurs in the centre of both graphs because of the local instability in the placement of Synechocystis PCC 6803. 
Table I: Genome features of obligate intracellular symbiotic and parasitic bacteria

\begin{tabular}{|c|c|c|c|c|c|c|c|}
\hline Species & $\begin{array}{l}\text { Genome size } \\
\text { (Mbp) }\end{array}$ & $\begin{array}{l}\text { AT-content } \\
\text { genome (\%) }\end{array}$ & $\begin{array}{l}\text { AT-content I6S } \\
\text { rDNA (\%) }\end{array}$ & $\begin{array}{l}\text { ATcontent Ist } \\
\text { codon }\end{array}$ & $\begin{array}{l}\text { ATcontent } 2 \text { nd } \\
\text { codon }\end{array}$ & $\begin{array}{l}\text { ATcontent } 3 r d \\
\text { codon }\end{array}$ & Ref. \\
\hline $\begin{array}{l}\text { Buchnera } \\
\text { aphidicola APS }\end{array}$ & 0.66 & 73.7 & 49.86 & 62.62 & 69.41 & 85.77 & [39] \\
\hline $\begin{array}{l}\text { Candidatus } \\
\text { Blochmannia } \\
\text { floridanus }\end{array}$ & 0.71 & 72.6 & 52.89 & 61.33 & 68.08 & 83.95 & [7] \\
\hline Carsonella ruddii & 0.16 & 83.4 & 64.15 & 80.26 & 79.61 & 92.22 & [9] \\
\hline $\begin{array}{l}\text { Chlamydia } \\
\text { trachomatis D/ } \\
\text { UW-3/CX }\end{array}$ & 1.04 & 58.7 & 48.58 & 48.39 & 61.17 & 65.52 & [40] \\
\hline $\begin{array}{l}\text { Rickettsia } \\
\text { prowazekii } \\
\text { Madrid E }\end{array}$ & 1.11 & 71.0 & 49.5 & 58.95 & 68.24 & 81.53 & [22] \\
\hline $\begin{array}{l}\text { Sodalis } \\
\text { glossinidius }\end{array}$ & 4.17 & 45.3 & 45.29 & 38.83 & 57.86 & 34.73 & {$[12]$} \\
\hline $\begin{array}{l}\text { Wigglesworthia } \\
\text { glossinidia }\end{array}$ & 0.7 & 77.5 & 51.19 & 69.12 & 71.84 & 88.12 & [38] \\
\hline $\begin{array}{l}\text { Spheroid } \\
\text { body }\end{array}$ & n.d. & $\begin{array}{c}62.8 \\
\text { (fragment) }\end{array}$ & 45.77 & 48.16 & 61.89 & 67.25 & this paper \\
\hline $\begin{array}{l}\text { Cyanothece sp. } \\
\text { ATCC } 51 / 42\end{array}$ & n.d. & 60.2 (fragment) & 45.42 & 49.16 & 61.40 & 66.81 & this paper \\
\hline
\end{tabular}

Genome size, AT-content and nucleotide composition of each codon position are indicated

(References: [7,9,12,22,38-40]). n.d.: not determined

\section{Gene deletions and modifications downstream the nif- region}

Conserved downstream of the nif-operon genes in Cyanothece ATCC 51142 and the spheroid body are genes encoding subunits of the NADH-dehydrogenase and ferredoxin as well as transporters for Fe and Mo (Figure 1). However, genes encoding photosynthetic proteins, which are precursors for cytochrome c6 (pet) ) and plastocyanin (pet $\mathrm{E})$, are absent in the spheroid body in this genome region. Interestingly, elsewhere in the genome, the photosystem II protein genes $p s b \mathrm{C}(\mathrm{CP} 43)$ and $p s b \mathrm{D}$ (D2) exist in an operon-like structure, similar to that of Cyanothece sp. CCY0110 and other cyanobacteria. However, in the spheroid body these genes are highly truncated or disrupted by several stop codons and thus exist as pseudogenes. This finding is consistent with the lack of photosynthetic activity previously reported for the spheroid body $[18,19]$.

Downstream of the highly conserved nif-gene region, other genetic modifications can be inferred. In Cyanothece ATCC 51142, and other closely related cyanobacteria the open reading frame (orf)cyl0012 is flanked by the genes for an iron-transporter (feoA) and a Mo-ABC-transporter $(\bmod C)$. This orf has been deleted in the spheroid body's genome (Figure 1). In this case the whole gene has been removed without trace of pseudogenisation or local sequence conservation. The orf identified as cyl0019 in Cyanothece ATCC 51142 has also been lost from the nifregion. In this species and other close relatives, this orf is flanked by two conserved orfs cyl0018 and cyl0020. In the spheroid body's genome, cyl0019 is deleted and the flanking orfs $c y l 0018$ and $c y l 0020$ are fused to give to the hypothetical protein sbl0010 (Figure 5). Not all deletions of genes in the spheroid body's genome have resulted in genome compaction as some genome regions appear replaced by non-coding regions as described in the following section.

\section{Extensive modifications lead to large non-coding regions in the spheroid body's genome}

A significant difference between the genome of Cyanothece sp. ATCC 51142 and that of the spheroid body is the extent of non-coding DNA stretches greater than $500 \mathrm{bp}$ (Figure 6). In the former there are three non-coding stretches at the nif locus (including downstream region). There are seven such regions in the spheroid body's genome fragment. One of these additional non-coding regions is located adjacent to the nif gene region, in a region of high synteny between both genomes. The noncoding regions of the spheroid body's genome are characterised by elevated levels of A and T nucleotides. Several pseudogenes are also located within these genome regions (Figure 1 and 6).

In Cyanothece ATCC 51142, the gene fdxIII and an orf coding for a conserved hypothetical protein (cyl0018) flank four open reading frames (cyl0014/vagC, cyl0015/pilT, $c y l 0016, c y l 0017 / v a p C)$. Three of these have highest similarity to virulence associated proteins (vap-proteins) and the fourth has greatest similarity with proteins containing a PIN-domain (Figure 1). In spheroid bodies, $f d x$ III and the 


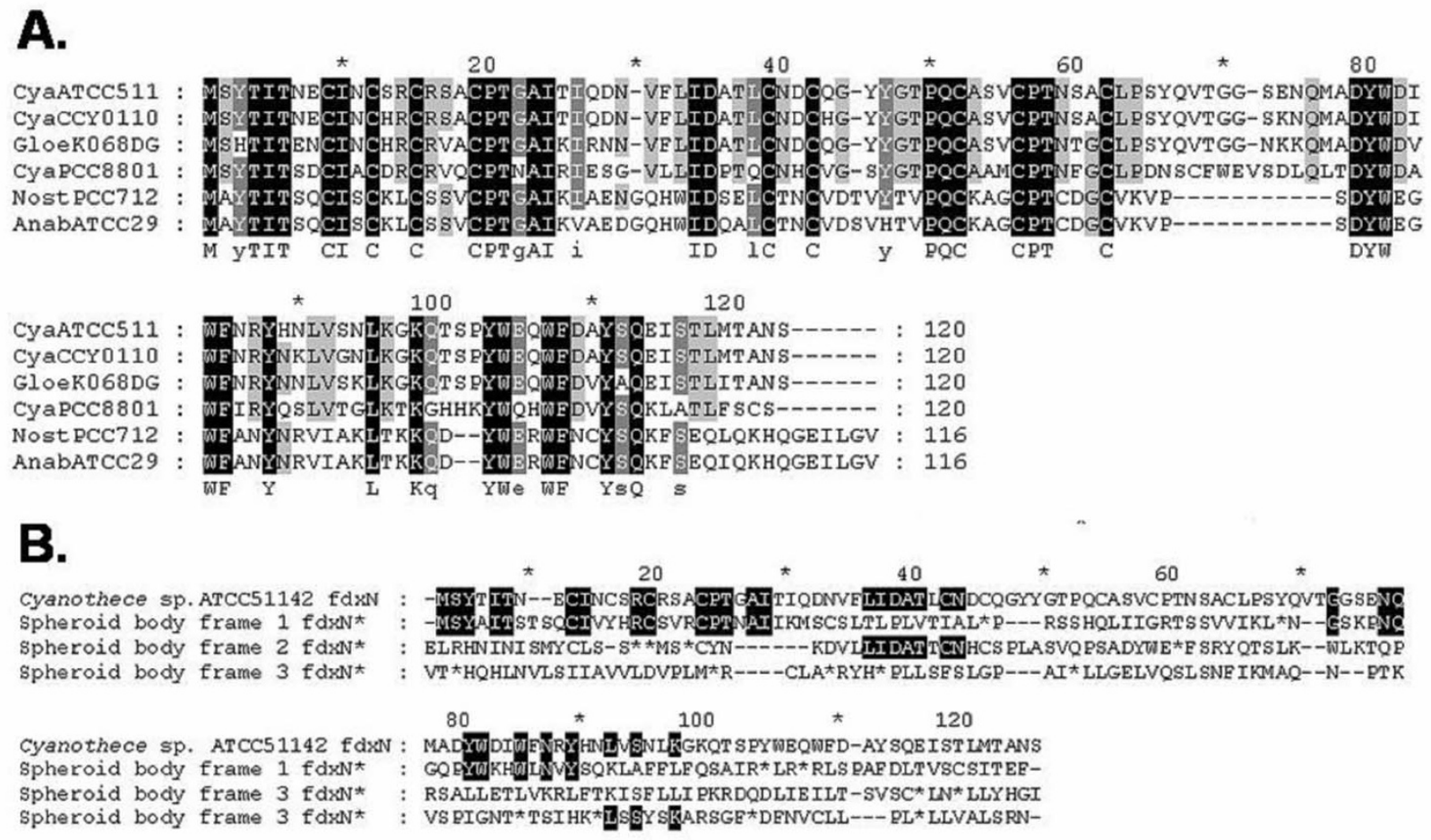

Figure 3

Analysis of Ferredoxin N. A. Multiple alignment of cyanobacterial FdxN proteins. Accession numbers: CyaATCC5I I42: AAW56985.I (Cyanothece sp. ATVV5I I42); CyaPCC880I: AAC33373.I (Cyanothece sp. PCC880I); CyaCCY0I I0: ZP 01727762.I (Cyanothece sp. CCY0I I0); GloeK068DGA: BAF47I48.I (Gloeothece sp. KO68DGA); NostPCC7I 20: AAA22005.I (Nostoc punctiforme PCC 7120); AnabATCC294I 3: YP 3244I3.I (Anabaena variabilis ATCC 294I3). B. Alignment of Cyanothece sp. ATCC5I | 42 FdxN protein with the spheroid body fdxN* pseudogene translated in 3 forward reading frames. Evidence of homology, at the level of amino acid similarity, is distributed across all 3 reading frames of the pseudogene, indicating multiple substitutions and single nucleotide deletion events.

homologue to $c y l 0018$ (as part of sbl0010, see above) frame a non-coding region of about 2000 bp which contains two pseudogenes: for a flavodoxin, long-chain hypothetical protein and an orf conserved in Crocosphaera sp.(CwatDRAFT_1967). This non coding region shows an increased AT-ratio of $73.5 \%$ (Figure 6). Its presence is the result of extensive genome modification which has led to the deletion of multiple genes. Unlike other regions where there has been gene deletion (e.g. as with cyl0012), this modification is not accompanied by the deletion of the genomic regions. It is possible that these regions might be the outcome of multiple mutations leading to the loss of genes, no longer recognizable as pseudogenes, with preservation of the genomic locus, which might be subsequently eliminated [22]. Because the whole genome size of spheroid bodies is not experimentally determined so far, it is not known if these modifications have lead to an overall decrease of the spheroid body genome size. As described in more detail in the discussion part, we used a bioinformatic model to predict the size of the whole sphe- roid body genome. Using this prediction, the genome size is estimated to be approximately $2,6 \mathrm{Mb}$.

To investigate whether intact copies of missing or pseudogenised genes (e.g. after genome rearrangement or gene duplication events) are present elsewhere in the spheroid body's genome, we performed PCR analysis with specific or degenerate primers for the cyanobacterial genes $f d x \mathrm{~N}$, petJ, psbC, cyl0012 and $c y l 0017$. As shown in Figure 7, no products were amplified using either spheroid body or $R$. gibba DNA as template, indicating that the identified (pseudo)genes do not have functional counter parts encoded elsewhere in the endosymbiont's genome.

\section{RecA and RecF are encoded by the spheroid body's genome}

The proteins RecA and RecF play an important role in recombinational repair of DNA as well as roles in other repair pathways like nucleotide excision (reviewed in [23]). From the study of insect-bacterium symbioses it is 
A.

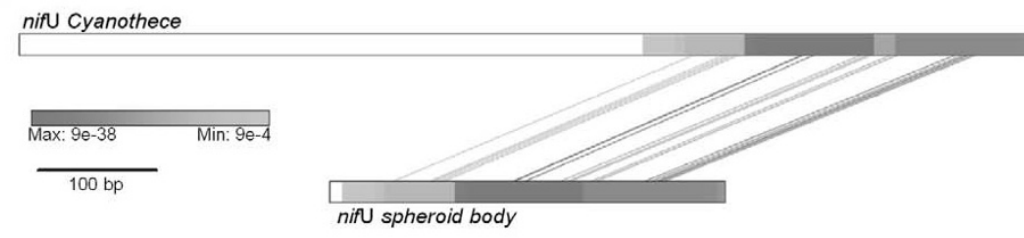

B.

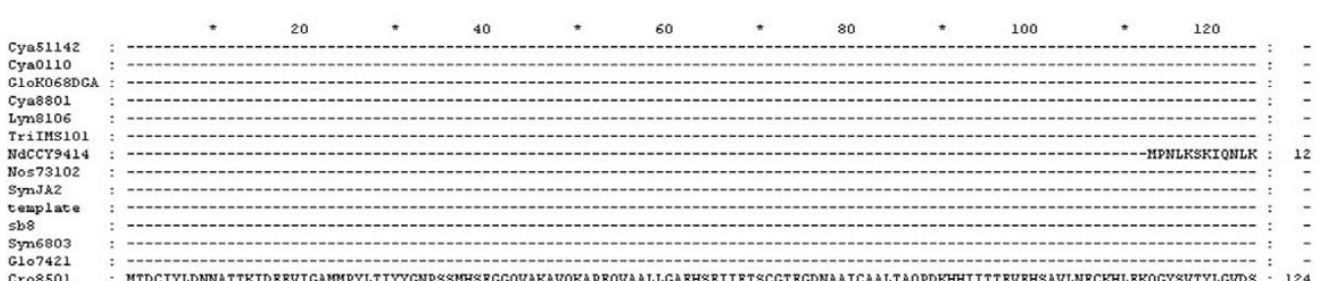

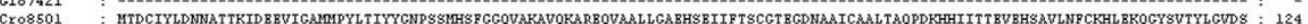

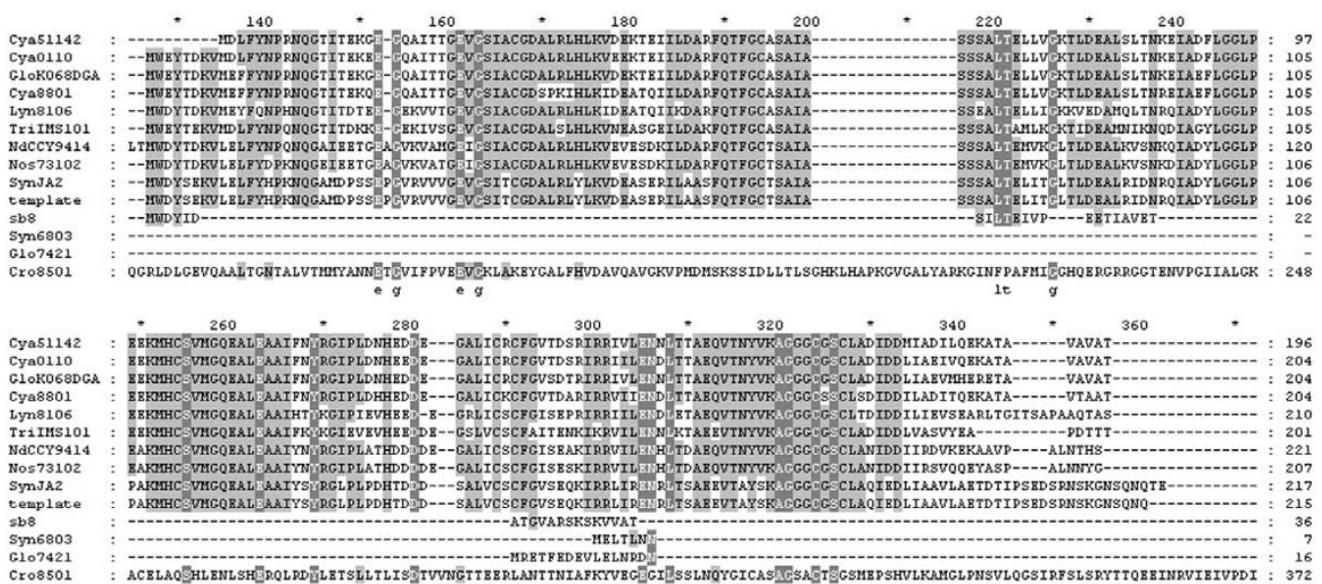

Cr08501 $:-16$

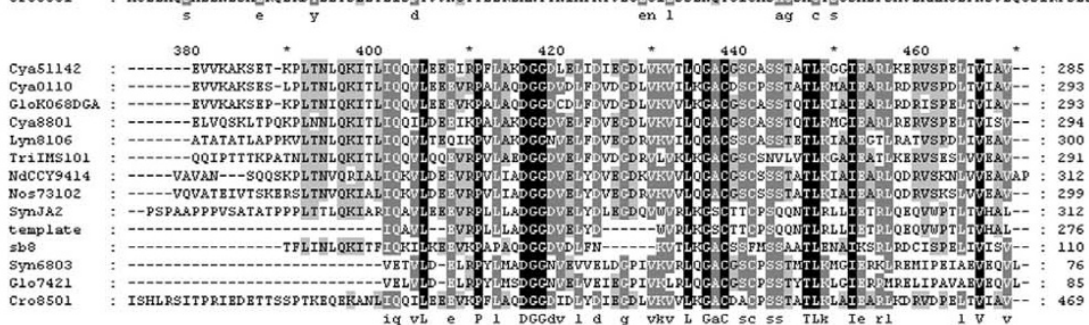

\section{Figure 4}

Truncation of nifU in spheroid bodies. A. GATA plot for Cyanothece and spheroid body nifU indicating conserved regions. GATaligner settings: Window size: 100; Match: 5; MisMatch:-4; Gap Creation:-10; Gap Extension:-4; Raw Score Cut Off: 92.0. GATAPlotter score settings: Max: I4I bits, expect 9E-38; Min: 28 bits, expect 9E-4. GATAPlotter scores are indicated. B. Multiple alignment of predicted amino acid sequences for NifU indicating an N-terminal truncation in the homologue from the endosymbiont. NifU accession numbers: Cya5 I I42: AAW56987.I (Cyanothece sp. ATCC 5I I42); Cya0 I I0: ZP_01727764.I (Cyanothece sp. CCY0II0); GloKO68DGA: BAF47I50.I (Gloeothece sp. KO68DGA); Cya880I: AAC3337I.I (Cyanothece sp. PCC 880I); Lyn8I06: ZP_0I620769.I (Lyngbya sp. PCC 8I06); TrilMSI0I: AAF82636.I (Trichodesmium sp. IMSI0I);

NdCCY9414: ZP_01628437.I (Nodularia spumigena CCY9414); Nos73 102: ZP_00I 123 I7.I (Nostoc punctiforme PCC 73 I02); SynJA2: YP_476679.I (Synechococcus sp. JA-2-3B'a(2-13)); sb8: AAW57048.I (spheroid body); Syn6803: NP_442853.I (Synechocystis sp. PCC6803); Glo742I: NP_925823.I (Gloeobacter violaceus PCC 742I); Cro850I: ZP_005I6385.I (Crocosphaera watsonii WH 850I). 


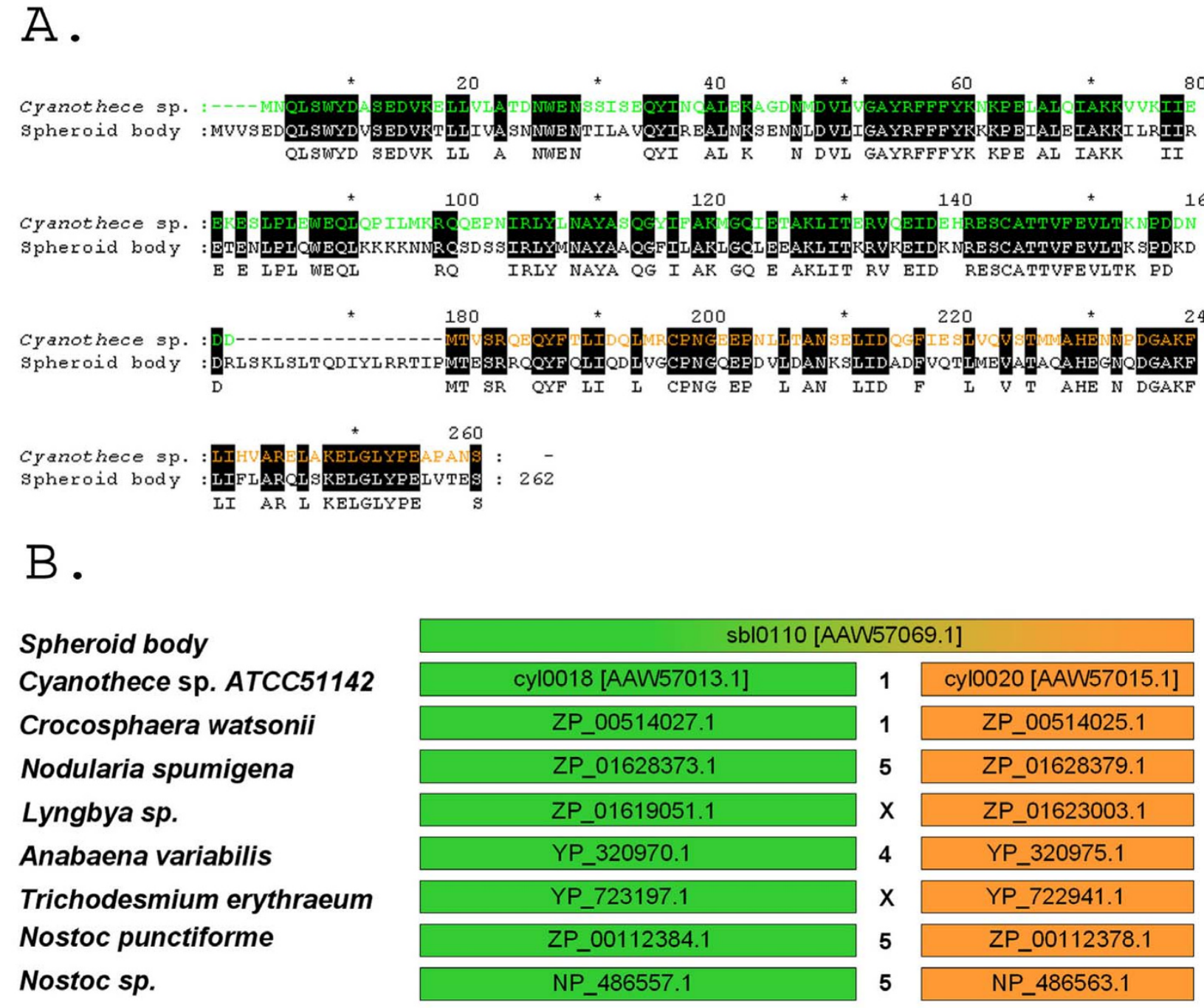

Figure 5

Spheroid body orf sbl00 I0 encodes a fusion protein derived from homologues of the Cyanothece sp.

ATCC5 I I 42 CyI00I 8 and Cyl0020 proteins. A. Alignment of predicted amino acid sequences for spheroid body protein SbI00IO and Cyanothece proteins Cyl00I8 and Cyl0020. Deletion in the endosymbiont genome of cyl00I9 in the creation of sbl00I0 can be inferred during reductive genome evolution. In SbI0010, homologues of Cyl00I8 and Cyl0020 have been conserved in full length and are separated by a 17 amino acid residues. Cyl00 I8: green, Cyl0020: orange, Sbl00 10: black. B.

Cyl00I 8 and Cyl0020 are highly conserved in cyanobacteria closely related to the spheroid body. They are aseparated by $1-5$ genes when they co-occur at the same locus, but in some cases they are encoded at different loci of the genome (indicated by $\mathrm{x})$.

known that gene loss and pseudogene creation is often associated with defects in $\mathrm{rec} \mathrm{A}$, and/or $\mathrm{rec} \mathrm{F}$ [10]. In order to investigate whether the observed inactivation and deletion of genes in the spheroid body's genome might be explained by defects in the bacterial repair systems, we searched the genomes of the spheroid body and of Cyanothece ATCC 51142 for recA and $r e c \mathrm{~F}$. As shown in Figure 8 , intact and highly conserved orfs can be identified in both genomes, indicating that repair pathways are active in the R. gibba endosymbiont.

\section{Accumulation of pseudogenes in the spheroid body's genome}

As already mentioned, by analysing the contiguous spheroid body's genome fragment (Figure 1) several pseudogenes could be readily identified, among them a mutated ferredoxin gene $\left(f d x \mathrm{~N}^{*}\right)$ within the nif-operon as well as other genes downstream of the nif-gene region. Additional pseudogenes for conserved cyanobacterial proteins were also identified when screening 140,000 bp of non-contiguous genome sequences from the spheroid bodies. These 


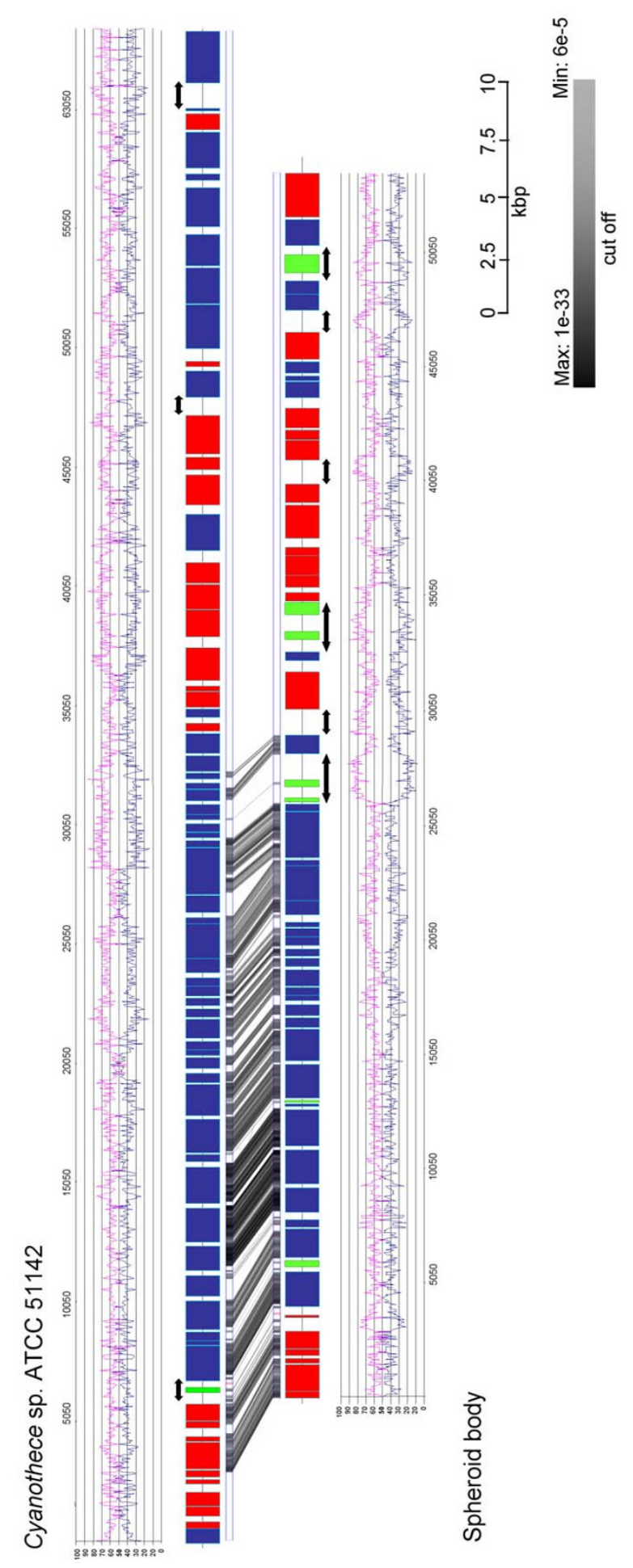

Figure 6

A/T-G/C frequencies in Cyanothece sp. ATCC 5 I I 42 and spheroid body genome fragments. A/T-G/C-plot for the genome region shown in Fig. I (red: AT; blue:G/C), indicating a high AT composition in the large non-coding regions (black arrows) of the spheroid body fragment. were found either via BLAST homology search (minimum e-value: 5e-10) or by analysis of spheroid body's genome regions corresponding to operons conserved in other cyanobacteria. Genetic regions with homologues in other species, divided into two fragments by one stopcodon or frameshift or just truncated were considered pseudogenes if the similar gene region was less than half the length of its homologue [12]. Deleterious mutations leading to pseudogenes were found in genes encoding proteins that affect cellular processes such as cell wall biosynthesis and transposon controlled genome rearrangement (summarized in Table 2). In Cyanothece sp. ATCC 51142, only one pseudogene was identified within the genome fragment that contained the nif-operon (Table 2). In contrast, six pseudogenes were found in the spheroid body's genome fragment. No pseudogenes were identified in the genome of Cyanothece sp. strain CCY0110 which is a very close relative of Cyanothece sp. ATCC 51142.

\section{Discussion}

An intriguing example of an obligate intracellular symbiotic interaction is the cyanobacterium-diatom symbiosis found in Rhopalodia gibba [18]. Here the symbiont (spheroid body) can fix nitrogen for its eukaryotic host, and we have hypothesised that this capacity has been a driving force for establishing the intracellular endosymbiotic relationship [17]. The spheroid body of Rhopalodia gibba provides an opportunity to investigate changes in endosymbiont physiology and genome evolution during adaptation of a symbiont to an intracellular environment.

Previous studies have reported changes in the genomes of bacteria following development of symbiotic relationships. In bacteria that are thought to have recently or transiently become symbiotic, changes include occurrence of multiple transposable elements and deletions of important components of recombinational DNA repair mechanisms [1]. In longer established symbiotic and parasitic eukaryote-bacterium interactions, significant gene losses have been observed, and these have been accompanied by reduction of genome size and generation of AT rich genomes $[3,6,24]$. Changes that have occurred in the spheroid body's genome can not be categorised as an obvious example of the former or latter relationship. For example, the spheroid body's genome encodes several transposase genes, all with disrupted reading frames, indicating that these are pseudogenes. This finding is consistent with stability of the diatom-spheroid body endosymbiosis and a long term host-endosymbiont interaction, which can be traced back to the Miocene [25]. Contrasting with the occurrence of transposase pseudogenes is evidence suggesting a functional DNA repair system in the spheroid body's genome. This is a finding more consistent with a relatively young endosymbiotic relationship. In nearly all intracellular bacteria studied to date, at 

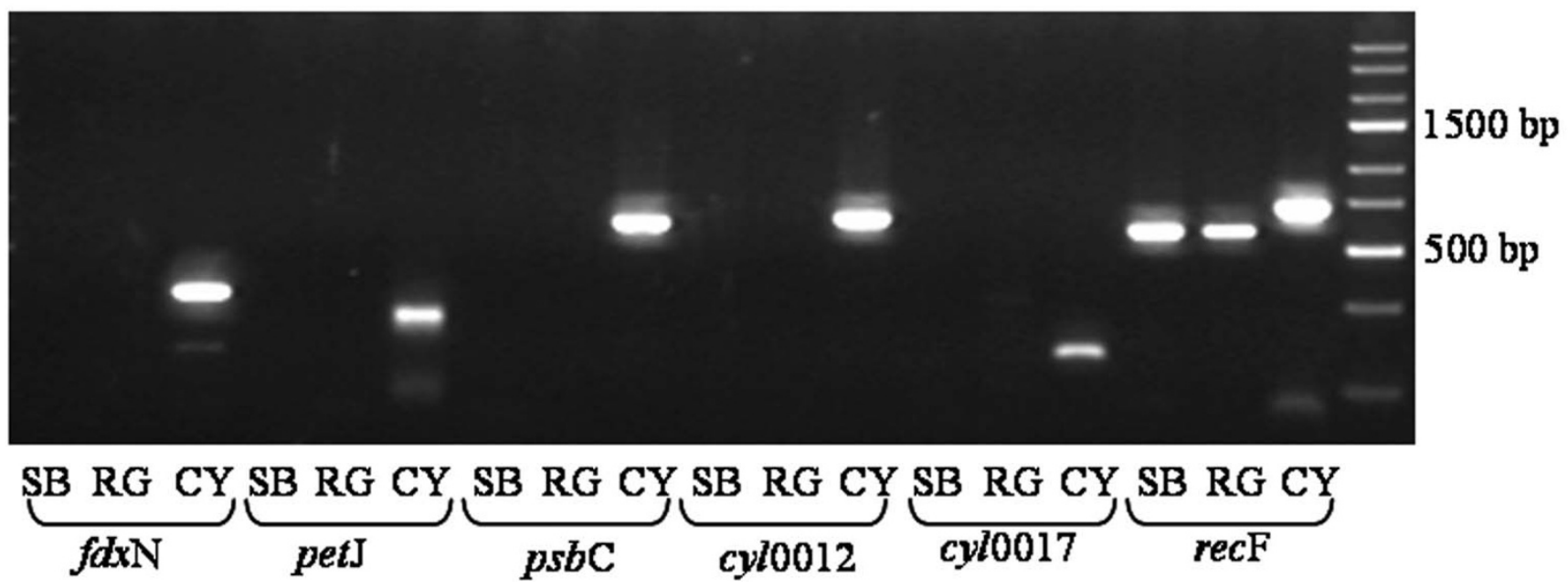

Figure 7

PCR analysis of missing or pseudogenisised genes. PCR analysis of Cyanothece sp. ATCC 5 II 42 (CY), R. gibba (RG) and spheroid body (SB) DNA. Amplification with primers specific for the cyanobacterial fdxN, pet], psbC, cyl00I 2 and cyl00I7 genes show that the analysed genes are not encoded in $R$. gibba and the endosymbiont's genome. CYrecF and SBrecF were used as positive controls. GeneRuler ${ }^{\mathrm{TM}}$ Express DNA Ladder (Fermentas) was used as molecular weight standard.

least one of the genes encoding the DNA repair proteins RecA and RecF has been eliminated. It is thought this might be necessary to facilitate restructuring of the symbiont genome (for the exception see [12]). In the spheroid body's genome both recA and recF are present and have intact open reading frames. Thus the genome modifications that we report for the spheroid body's genome have all occurred against a background of a presumably intact DNA repair system. These modifications suggest that selective pressure for certain genes has changed upon establishment of the interaction, and the challenge is to attempt to understand the potential relevance of these for necessary and redundant functions in an obligate endosymbiotic relationship. For example, gene truncations as detected e.g. in nifu, would remove genes redundant for diazotrophic growth [26], and such deletion might be an early event in genome reduction of the symbiont. A subsequent or perhaps parallel step would include the inactivation of genes whose gene products are no longer needed for the initial symbiotic association. For this to occur, various different possible scenarios could be hypothesized: inactivation of genes by deleterious mutations resulting in the accumulation of pseudogenes or loss of genome fragments by deletion of larger DNA portions via rearrangements [27]. Another hypothesis posits a "domino-effect" of initial pseudogenisation triggering subsequent largescale gene loss [28]. In this scenario, random pseudogenisation might lead to the inactivation of a pathway due to mutation of a single essential factor, followed by largescale deletion of other genes involved in this pathway. In each case, the selective pressure would be different for genes coding for different functions, and loss would depend on whether function could be compensated by other genes in the endosymbiont or host cell genome. In the latter case, as in highly adapted interactions, signaldependent transport of the protein from the host cytoplasm to the endosymbiont would be necessary.

We detected several examples for the disruption of coding regions by mutations (Table 1 ), in which the original gene is still detectable by analysis of all three possible frames. This includes psuedogenisation of $f d x \mathrm{~N}\left(f d x \mathrm{~N}^{*}\right)$, a gene which has been found to be non essential for nitrogen fixation in Anabaena variablis [20] and several other genes on spheroid body's genome fragments that we have sequenced (Table 2, Figure 1). Such observations provide further evidence that pseudogenisation of genes, which are non-essential for endosymbiotic life-style, is an important feature in the early reductive genome evolution of obligate intracellular cyanobacteria. Gene loss through independent DNA deletion events could also be inferred in comparative analysis of the spheroid body's genome fragment; among these the deletion of factors conserved in diverse cyanobacterial lineages (cyl0012, cyl0019). Due to elimination of the immediate DNA region, these modifications have led to a localised increase in gene density. In one extreme, deletion has produced a fusion of nonadjacent genes on the endosymbionts genome (sbl0010). In other cases of gene deletion, genes have been removed and replaced with non-coding sequence that is much higher in AT-content than occurs in the coding regions (Figure 6). It is unclear whether this difference in compo- 
RecA

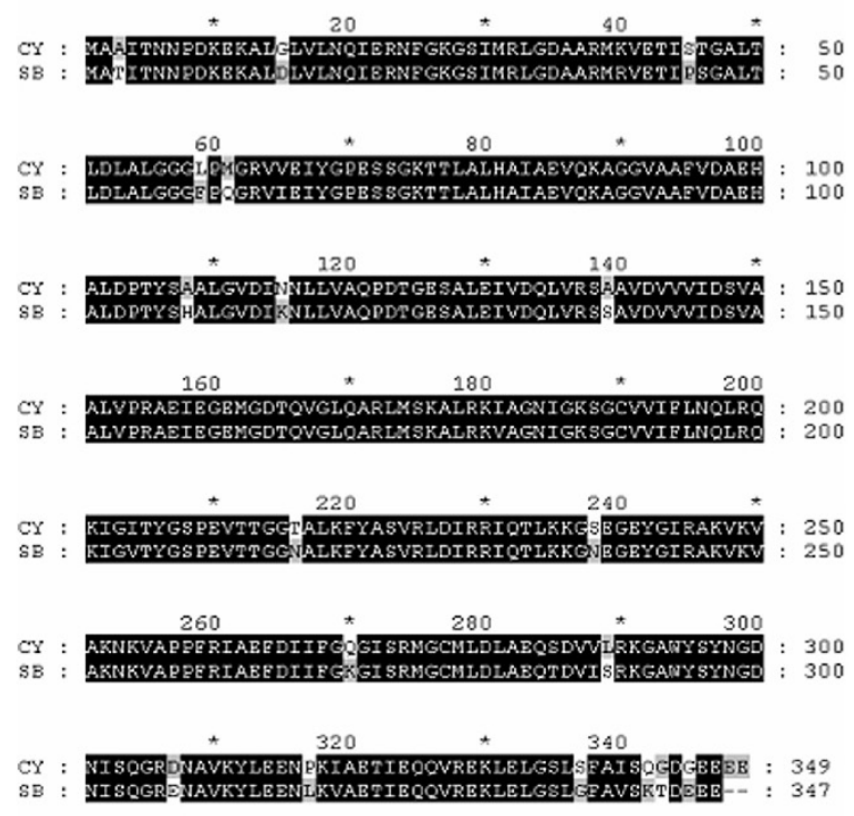

RecF
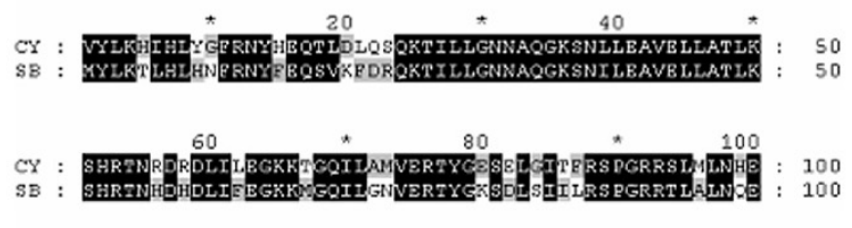

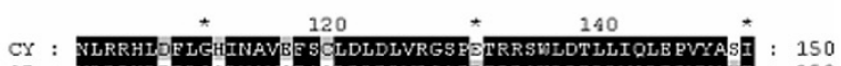

SE : VLRRHIEELGSLNAV SESLLDLDLVGSPDRRSWLDTLLVQLE PVYAHI : 150

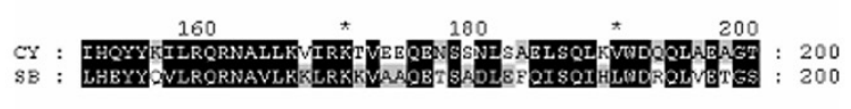

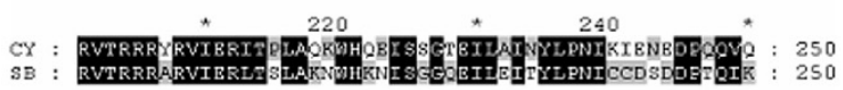

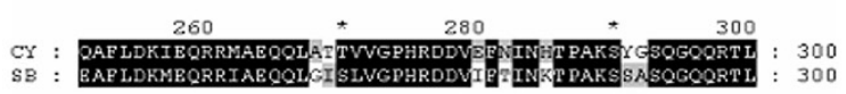

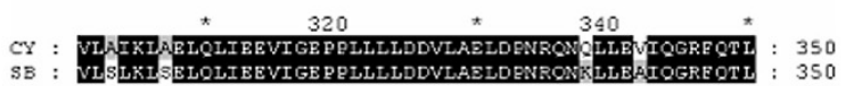

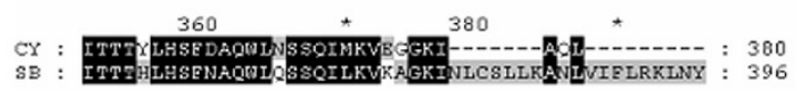

Figure 8

Pairwise alignments for RecA and for RecF. Alignment of RecA (left) and RecF (right) homologues from the spheroid body (SB) and Cyanothece sp. ATCC5II42 (CY) genomes. The spheroid body encodes complete full length recA and recF orfs. 
Table 2: Pseudogenes in all sequenced spheroid body genome fragments (totalling $192335 \mathrm{bp}$ ) and pseudogenes identified in the Cyanothece sp. ATCC 51142 genome region shown in Figure I (63344 bp)

\begin{tabular}{|c|c|c|c|c|c|}
\hline No. & Name & Best BlastX hit & Organism & Accession & e-value \\
\hline sbpl* & $f d x N^{*}$ & $\mathrm{FdxN}$ & Cyanothece sp. PCC 880I & AAC33373.1 & 0.070 \\
\hline $\operatorname{sbp} 2 *$ & fldA* & Flavodoxin, long chain & Crocosphaera watsonii WH $850 \mathrm{I}$ & $\underline{Z P \quad 00515759.1}$ & $2 e-05$ \\
\hline $\operatorname{sbp} 3 *$ & $s b p 3^{*}$ & hypothetical protein CwatDRAFT_1967 & Crocosphaera watsonii WH $850 \mathrm{I}$ & $\underline{Z P \quad 00517249.1}$ & $7 e-05$ \\
\hline $\operatorname{sbp} 4 *$ & $s b p 4^{*}$ & hemolysin & Cyanothece sp. CCYOIIO & $\underline{Z P} 01728583.1$ & $3 e-09$ \\
\hline $\operatorname{sbp} 5^{*}$ & $\operatorname{sbp} 5^{*}$ & Putative esterase & Crocosphaera watsonii WH $850 \mathrm{I}$ & $\underline{Z P \quad 0051494 I .1}$ & $6 e-16$ \\
\hline sbp6* & $u b i H^{*}$ & $\begin{array}{l}\text { COG0654: 2-polyprenyl-6-methoxyphenol } \\
\text { hydroxylase and related FAD-dependent } \\
\text { oxidoreductases }\end{array}$ & Nostoc punctiforme PCC 73102 & $\underline{Z P 00110350.1}$ & $2 \mathrm{e}-17$ \\
\hline $\operatorname{sbp} 7^{*}$ & $s b p 7^{*}$ & hypothetical protein CY0II0_23906 & Cyanothece sp. CCYOIIO & $\underline{Z P} 01727952.1$ & $5 e-09$ \\
\hline $\operatorname{sbp} 8 *$ & $\operatorname{sbp} 8^{*}$ & hypothetical protein CYOIIO_II 227 & Cyanothece sp. CCYOIIO & ZP 01730915.1 & le-18 \\
\hline $\operatorname{sbp} 9 *$ & $s b p 9 *$ & $\begin{array}{l}\text { Peptidase S49, SppA } 67 \text { kDa type:Peptidase S49, } \\
\text { SppA }\end{array}$ & Crocosphaera watsonii WH $850 \mathrm{I}$ & $\underline{Z P} 00518433.1$ & $3 e-09$ \\
\hline sbpl0* & $\operatorname{sbpl0*}$ & GDP-D-mannose dehydratase & Cyanothece sp. CCYOIIO & $\underline{\text { ZP } 01730804.1}$ & $|e-| 3 \mid$ \\
\hline sbpll* & $\operatorname{sbp} \mid I^{*}$ & NAD-dependent epimerase/dehydratase & Crocosphaera watsonii WH $850 \mathrm{I}$ & $\underline{Z P \quad 00513970.1}$ & $5 e-103$ \\
\hline $\operatorname{sbp} 12^{*}$ & $\operatorname{sbp} 12 *$ & hypothetical protein CwatDRAFT_2668 & Crocosphaera watsonii WH $850 \mathrm{I}$ & $\underline{Z P \quad 005171115.1}$ & $3 e-10$ \\
\hline sbpl3* & uma4* & Uma4 (transposase homolog) & Microcystis aeruginosa PCC 7806 & AFI83408 I5 & $4 e-05$ \\
\hline sbpl4* & $\operatorname{sbpl} 4^{*}$ & hypothetical protein CY0IIO_27355 & Cyanothece sp. CCYOIIO & ZP 01729498.1 & $9 e-07$ \\
\hline sbpl5* & $\operatorname{sbp} 15^{*}$ & thioredoxin reductase & Lyngbya sp. PCC 8106 & $\overline{Z P} 01622501.1$ & $8 e-88$ \\
\hline sbpl6* & $\operatorname{sbpl6*}$ & putative transposase & Lyngbya sp. PCC 8106 & $\overline{Z P 01618921.1}$ & $6 e-65$ \\
\hline $\operatorname{sbp} 17^{*}$ & IldP* & hypothetical protein DSY226I & Desulfitobacterium hafniense Y5I & YP 518494.1 & |e-I4 \\
\hline sbpl8* & $\operatorname{sbp} 19 *$ & $\mathrm{Mg}$ chelatase-related protein & Cyanothece sp. CCYOIIO & $\overline{Z P \quad 01732243.1}$ & $2 e-06$ \\
\hline sbpl9* & $s b p 20^{*}$ & hypothetical protein CY0II0_073I4 & Cyanothece sp. CCYOIIO & ZP 01728735.1 & $2 e-103$ \\
\hline sbp20* & $r s b W *$ & $\begin{array}{l}\text { Putative Anti-Sigma regulatory factor (Ser/Thr } \\
\text { protein kinase) }\end{array}$ & Cyanothece sp. CCYOIIO & $\underline{Z P \quad 01726436.1}$ & 0.011 \\
\hline $\operatorname{sbp} 21 *$ & $s b p 22 *$ & $\begin{array}{l}\text { Anti-Sigma-factor antagonist (STAS) and sugar } \\
\text { transfersase }\end{array}$ & Cyanothece sp. CCYOIIO & ZP 01726435.1 & $7 e-53$ \\
\hline $\operatorname{sbp} 22^{*}$ & $\operatorname{sbp} 23 *$ & transposase & Nostoc sp. PCC 7120 & NP 484634.I & $4 e-20$ \\
\hline sbp23* & $I_{R} K^{*}$ & hypothetical protein CYOIIO_I2822 & Cyanothece sp. CCYOIIO & $\underline{Z P} \quad 01729432.1$ & $5 e-28$ \\
\hline sbp24* & orfAB* & orfAB & Nostoc sp. PCC 7120 & AAC97588.I & $3 e-05$ \\
\hline $\operatorname{sbp} 25^{*}$ & $\operatorname{sbp} 26^{*}$ & Protein of unknown function DUF820 & Crocosphaera watsonii WH $850 \mathrm{I}$ & $\underline{Z P \quad 00514570.1}$ & $2 e-13$ \\
\hline sbp26* & $\operatorname{sbp} 27^{*}$ & probable sulfotransferase & Cyanothece sp. CCYOIIO & $\overline{Z P \quad 01732095.1}$ & $4 e-50$ \\
\hline sbp27* & $\operatorname{sbp} 28^{*}$ & COG3464: Transposase and inactivated derivatives & Nostoc punctiforme PCC 73102 & $\overline{Z P \quad 00108066.1}$ & $2 e-11$ \\
\hline sbp28* & s_TKc* & serine/threonine protein kinase & Cyanothece sp. CCYOIIO & $\underline{Z P \quad 01731251.1}$ & $4 e-26$ \\
\hline sbp29* & $s b p 30 *$ & hypothetical protein CwatDRAFT_4770 & Crocosphaera watsonii WH $850 \mathrm{I}$ & ZP 00515299.1 & $8 e-07$ \\
\hline $\operatorname{sbp} 30^{*}$ & $\operatorname{ded} A^{*}$ & DedA & Lyngbya sp. PCC 8106 & $\underline{Z P \quad 01623614.1}$ & $2 e-53$ \\
\hline $\operatorname{sbp} 31 *$ & $\operatorname{sbp} 32^{*}$ & hypothetical protein CY0II0_03639 & Cyanothece sp. CCYOIIO & $\overline{Z P \quad 01727528.1}$ & $5 e-06$ \\
\hline $\operatorname{sbp} 32 *$ & $\mathrm{me} / \mathrm{B} *$ & Sodium:galactoside symporter & Crocosphaera watsonii WH $850 \mathrm{I}$ & $\underline{Z P \quad 00517271.1}$ & $5 e-22$ \\
\hline sbp33* & $p s b C^{*}$ & photosystem II CP43 protein & Synechocystis sp. PCC 6803 & NP 441119.1 & 1,0 \\
\hline sbp34* & psbD* & photosystem II D2 protein & Cyanothece sp. CCYOIIO & ZP 01728138.1 & $5 e-05$ \\
\hline cypl* & cypl* & hypothetical protein CY0II0_22382 & Cyanothece sp. CCYOIIO & $\underline{Z P \quad 01727759.1}$ & $2 e-11$ \\
\hline
\end{tabular}

Pseudogenes within the nif-operon and downstream regions (genome regions shown in Figure I) are indicated in grey.

sition reflects a shift in substitutional bias favouring A and T residues, and/or whether an existing bias becomes more apparent in de-novo regions that are under reduced structural/function constraint. In either event, the existence of these AT rich non-coding regions suggests that pseudogenisation and DNA deletion are not inevitably linked events in a sequential process of degenerative genome evolution in spheroid bodies. However, non-coding regions are rare in genomes of free-living bacteria. Since DNA can be introduced in several ways into prokaryotic genomes, their compactness is maintained by the deletion of harmful DNA. Given the intracellular existence of spheroid bodies, it is possible that their genome is less exposed and less susceptible to introductions of foreign DNA through mechanisms of horizontal gene transfer and lysogenic bacteriphages in comparison to those of free-living bacteria. If so, processes excluding non-coding DNA and pseudogenes from the spheroid body's genome may well be less efficient than those operating in free living bacteria. Such a hypothesis might help explain the greater extent of non-coding DNA and pseudogenised genes in the spheroid body's genome. Increased mutation rates, thought to be associated with reductive genome evolution would contribute to accumulation of these genome features [29]. The genome modifications observed in the spheroid body are in some respects comparable to those of Sodalis glossinidius. A large fraction (49\%) of the Sodalis genome is composed of non-coding DNA that has accom- 
panied reductive genome evolution. Moreover, the Sodalis chromosome contains many unusual pseudogenes [12]. The spheroid body's genome differs from Sodalis with respect to their generally higher AT-content.

The diverse features of reductive genome evolution in obligate intracellular symbionts (and pathogens) include a significant reduction of overall genome size in these organisms. However, the experimental determination of the spheroid body's genome size using standard molecular techniques is difficult due to the extreme stability of the host-spheroid body interaction and the limited amount of intact and purified endosymbionts that can be obtained from R.gibba. Recently in a study on the dynamics of reductive evolution, exponential relationships were inferred between genome size and SSU rDNA GC-content in mitochondria, free-living and obligate intracellular bacteria [30]. Based on the model these authors propose, and using 16S sequence data previously published [18], we have estimated that the genome size of spheroid bodies is approximately $2.6 \mathrm{Mb}$. The genome size of free-living Cyanothece sp. CCY0110 is $5.8 \mathrm{Mb}$. Hence if our estimate of the spheroid body's genome size is accurate, this estimate suggests that reduction has produced a genome currently similar in size to that of Synechococcus $(2.2-2.6 \mathrm{Mb})$, and may indicate that the endosymbiosis is still at an early state of development.

Our comparative analyses of spheroid body's genome fosmid sequences indicate that the photosynthetic genes $p s b \mathrm{C}$ and $p s b \mathrm{D}$ have been inactivated by mutation in the endosymbiont genome. These gene products are essential factors in the photosynthetic light reaction of photosystem II [31]. According to the "domino-effect" hypothesis [28] initial deletion of components such as PsbC and PsbD is expected to lead to mass deletion of other genes involved in photosynthetic light reactions. Consistent with this prediction, additional photosynthetic factors that occur in closely related cyanobacteria are either absent (e.g. the cytochrome PetJ and the plastocyanine precursor PetE) or appear as a non functional pseudogene (e.g. the flavodoxin fldA*) in the spheroid body's genome.

Aside from gene loss resulting from reductive genome evolution, the absence of certain genes within the analysed genome region could also be explained by gene duplications or rearrangements. Without the complete sequence of the spheroid body's genome we can not exclude the possibility that following duplication, pseudogenisation has affected copies of some genes within the analysed genome region, while functional copies are retained elsewhere. However, the phenotypic loss of photosynthetic pigmentation indicates a complete loss of at least one essential factor of photosynthesis in the endosymbiont's genome. In addition, PCR analysis did not identify intact $p s b C$ and $p s b D$ genes present anywhere else in the spheroid body's genome (Figure 7).

The diverse modifications in the analysed spheroid body's genome fragment are not equally distributed over the whole sequence but accumulate downstream of the conserved nif gene region (Figure 1 and 5). This skewed distribution of degenerative modifications possibly reflects purifying selection acting across this genome region during the molecular adaptation process [32]. Aside from the mutation of $f d x \mathrm{~N}^{*}$ - a protein unimportant for nitrogen fixation -and the truncation of nif $\mathrm{U}$, all proteins for nitrogen fixation are conserved in the region without signs of degenerative genome evolution. This conservation of nif genes is consistent with the hypothesis that molecular nitrogen fixation has been an important driving force for the endosymbiotic interaction.

It can be expected that endosymbiont and host biochemistry will change with the development of the symbiotic interaction. Genes whose products become superfluous for symbiont-host coexistence are expected targets for mutation. At earlier stages of accumulation of deleterious mutation, holomologues will still be identifiable by BLAST homology searches. Table 2 lists many pseudogenes that may fit this category.

\section{Conclusion}

A diverse range of genetic modifications have occurred in the genome of $R$. gibba spheroid bodies and these would compromise the ability of the endosymbiont to exist as a free-living cyanobacterium, thereby confirming their suspected obligate status. Our findings provide insight into the genome evolution of a nitrogen-fixing endosymbiontic cyanobacterium living within a unicellular eukaryotic host. These are of special importance, as past inferences about processes of reductive genome evolution have mainly been based on the study of insect-bacteria interactions. In these, the symbionts reside within special cells or organs and thus their genomes may haven been subject to selection pressures different from those acting on the genomes of intracellular endosymbionts found in unicellular host organisms. Further analysis of the whole spheroid body's genome and comparison with free-living cyanobacteria will provide additional important information on the age of the interaction and the importance of different molecular processes and genetic modifications. Since the spheroid body is derived from cyanobacteriallike ancestors, the interaction could also serve as useful model system for understanding early events in the evolution of chloroplast genomes. 


\section{Methods \\ Symbiont Isolation and Purification}

Spheroid bodies of $R$. gibba were isolated and DNA was purified as described by Prechtl [18]. Cyanothece sp. ATCC 51142 genomic DNA was isolated using standard procedures [33].

\section{Construction of gDNA libraries}

Fosmid libraries of spheroid bodies and Cyanothece sp. ATCC 51142 were prepared using the fosmid library construction kit (Epicentre). After physical shearing, the DNA was blunt-end repaired and gel-fractionated to a fragment size of approximately $40 \mathrm{kbp}$ as described by the manufacturer. Insert-DNA was ligated in the pCC1-Fos ${ }^{\mathrm{TM}}$ vector, constructs were in vitro packaged into phage particles and transfected into Escherichia coli $\mathrm{EPI} 300^{\mathrm{TM}}-\mathrm{T} 1^{\mathrm{R}}$.

\section{Screening for nif-gene region, $\operatorname{rec} A$ and recF}

Screening for clones containing the nitrogen fixing operon and flanking sequences and clones containing the recA and $r e c \mathrm{~F}$ genes was performed using colony-PCR screening with oligonucleotides specific for spheroid body and the Cyanothece nif $\mathrm{D}-$, recA- and recF-gene, respectively. Primer sequences were SBnifD_uni: 5'-CGG ACA AAG AAA ACG CAG AAT TTG-3', SBnifD_rev: 5'-CAG AAC GTC ATC ACA CTG TTT TTG-3', CynifD_uni: 5'-CCG TCA CGT TGT TCC TGC TTT C-3' CynifD_rev: 5'-CCA AGG GGT GCC AAT TAA TCC C-3', SBrecA_uni: 5'-CTA CTC TCG CTC TCC ATG CGA TTG-3', SBrecA_rev: 5'-CGG CGA ATA TCT AAA CGG ACT GAG-3', CyrecA_uni: 5'GAT CGC AGA GGT GCA AAA GGC TG-3', CyrecA_rev: 5'CAG TTC CTC CGG TGG TGA CTT C-3', SBrecF_uni: 5'TCG GAC CTC AGC ATT ATC-3', SBrecF_rev: 5'-TCG ATG AGGTCC TAC TAA GC-3', CyrecF_uni: 5'-GCC GTC GAA TTA TTA GCA ACC C-3' and CyrecF_rev: 5'-GAA TTC GAC ATC ATC TCG ATG GG-3'. Clones for the upstream and downstream regions of positive nif-fosmids were obtained using the primers F13A12/3_uni: 5'-GAA CTC TAC AAT ACA GAT TAA CCG C-3', F13A12/3_rev: 5'-CAC TAA TCC ATC TAG ATT AGC CAC T-3', F13A12/5_uni: 5'-GGG CAT TCC AGA ATT AGA AGT AGG-3' and F13A12/5_rev: 5'CTG TAG CCA AGC CAA AGT CGT TAT G-3' for the spheroid bodies and F4D10/5_uni: 5'-CAA GCT GTC TTT GGA CAA AAG-3', F4D10/5_rev: 5'-CGT TGA AGG TTT CCT CAA AAC-3', F4D10/3_uni: 5'-GAT ATC GTT GAA ACC TAT CGA G-3' and F4D10/3_rev: 5'-GAA TGT TAG GAC GAG CAA AAG G-3'. PCR reactions were performed using standard procedures.

\section{Cloning of positive fosmids}

Preparation of fosmids and other DNA was performed according to standard protocols [33]. For subcloning, fosmid DNA was physically sheared by sonification. After blunt-end repair using the DNA Terminator Kit (Lucigen) and size fractionation by gel electrophoresis, fragments between 1000 and 1500 bp were isolated. The fragments were ligated in the pEZSeq ${ }^{\mathrm{TM}}$-vector (Lucigen) as described by the manufacturer and used to transform E. coli XL1blue MRF' cells. Sequencing of shotgun plasmids was carried out using cycle sequencing with 700 and $800 \mathrm{~nm}$ fluorescent labeled oligonucleotides and the $\mathrm{LICOR}^{\mathrm{TM}}$ sequencing system. 5'- and 3'-end sequencing of positive fosmid clones was performed using the primers M13 (700): 5'GTA AAA CGA CGG CCA GT-3' and a modified $\mathrm{pCC}^{\mathrm{TM}} /$ pEpiFOS $^{\text {тм }}$ RP-2 (800): 5'-GCC AAG CTA TTT AGG TGA G$3^{\prime}$. Shotgun inserts in the $\mathrm{pEZSeq}{ }^{\mathrm{TM}}$-vector were sequenced with M13_for: 5'-AGC GGA TAA CAA TTT CAC ACA GGA3' and M13_rev: 5'-CGC CAG GGT TTT CCC AGT CAC GAC-3'

\section{PCR analysis of missing or pseudogenised genes in the spheroid body's genome}

PCR analysis of missing or pseudogenised genes in the spheroid body's genome was performed with primers specific for cyanobacterial $f d x \mathrm{~N}$, petJ, psbC, cyl0012 and cyl0017 genes. Primer sequences were fdxNuni: 5'-AGT TAC ACT ATC ACC AAT G-3', fdxNrev: 5'-ATT TCT TGG GAG TAA GCA TC-3', CYpetJuni: 5'-ATG AAA AGA TTA TTG TCC CT-3', CYpetJrev: 5'-TGC TTG ACT TAA RAC ATA AG-3', psbCuni: 5'-ACG TAG TTA AAG GAG TTA ACG-3', psbCrev: 5'-TTC GGC TAT CTG CTG AAA GC-3', CY0012uni: 5'-CCT CTC AAC TTA GCC ATT AG-3', CY0012rev: 5'-AAG CTT TGC TGT GTA GAA AC-3', CY0017uni: 5'-ATN RTN GGN TGY MGN AAY AA-3' and CY0017rev: 5'-GCD ATN ARN SHR TCN GGD AT-3'. CYrecF and SBrecF were used as positive controls, with the same primers used for the fosmid screening experiments. PCR reactions were performed using standard procedures.

\section{Sequence homology determinations and annotation}

We assembled, finished and annotated sequences using the Sequencher [34] and Sequin software to allocate data and facilitate annotation. Identification of orfs was accomplished using BLAST analyses. Comparison of genome fragments was performed using BLAST analysis and the GATA tool for comparative sequence analysis [35]. Pseudogenes with one or more mutations were identified by BLAST searches or direct analysis of all open reading frames. Genome fragments of both Cyanothece sp. ATCC 51142 and $R$. gibba spheroid bodies are annotated in GenBANK under the accession numbers AY728386 and AY728387, respectively. Genes and orfs identified in both organisms were named according to BLASTp protein homologue names. Those orfs with homology to uncharacterised conserved hypothetical proteins and hypothetical orfs without any BLAST hit are numbered and referred as conserved hypothetical proteins and hypothetical proteins, respectively. Orfs oriented in the forward or reverse direction of the analysed fragment are named $c y l$ or $c y r$ for 
Cyanothece, sbl or $s b r$ for spheroid bodies and follow consecutive numbering (see additional files 1 and 2 ).

\section{Phylogenetic tree building}

Orthologues for spheroid body proteins greater than 200 amino acids from the genome region shown in Figure 1 were identified in closely related cyanobacteria using BLAST. These were aligned using CLUSTALX [34] and edited so that only conserved blocks of residues were used for evolutionary tree building. PHYML [36] was used to build maximum likelihood trees, assuming a JTT model of substitution and non-parametric bootstrapping (100 replicates). Strict consensus trees were built for each gene using the 100 gene trees produced from bootstrap replicates. SplitsTree 4.0 [37] was then used to build the supernetworks shown in Figure 2. Some proteins greater than 200 amino acids in length did not produce resolved strict consensus trees or were problematic for other reasons and these were omitted from the analysis (these included DapF, PyrF, Sbr0016, Sbl0019, FeoB1, NifP and Sbl0010). Protein sequences, additional to those reported in the present study and used for phylogenetic anlayses were those inferred from nucleotide sequences in both complete and unfinished genome projects. Genbank accession numbers for these are: Cyanothece sp. CCY0110 (AAXW00000000), Crocosphaera watsonii WH 8501 (AADV00000000), Nodularia spumigena CCY9414 (NZ AAVW00000000), Nostoc punctiforme PCC 73102 (NZ_AAAY00000000), Nostoc sp. PCC 7120 (NC_003272), Anabaena variabilis ATCC 29413 (NC_007413), Lyngbya sp. PCC 8106 (NZ AAVU00000000), Trichodesmium erythraeum IMS101 (NC_008312), Synechocystis sp. PCC 6803 (NC_000911).

\section{Authors' contributions}

CK and CV performed the molecular studies, sequences alignments and drafted the manuscript. PL participated in drafting the manuscript and performed the phylogenetic analyses. UGM conceived of the study, participated in its design and coordination and helped to draft the manuscript. All authors read and approved the final manuscript.

\section{Additional material}

\section{Additional File 1}

BlastP analysis of identified and annotated orfs of Cyanothece sp. ATCC 51142 (accession number AY728386). The table provides information on all annotated orfs of the analysed Cyanothece sp. ATCC 51142 genome fragment.

Click here for file

[http://www.biomedcentral.com/content/supplementary/14712148-8-30-S1.doc]

\section{Additional File 2}

BlastP analysis of identified and annotated orfs of Rhopalodia gibba spheroid bodies (accession number AY728387). The table provides information on all annotated orfs of the analysed spheroid body fragment genome fragment.

Click here for file

[http://www.biomedcentral.com/content/supplementary/14712148-8-30-S2.doc]

\section{Acknowledgements}

Our work is supported by the Deutsche Forschungsgemeinschaft (SFB 395, TP B9), the Alexander von Humboldt Foundation, and New Zealand Marsden Fund.

\section{References}

I. Moran NA, Wernegreen J]: Lifestyle evolution in symbiotic bacteria: insights from genomics. Trends in Ecology \& Evolution 2000, I5:321-326

2. Ochman H, Moran NA: Genes lost and genes found: Evolution of bacterial pathogenesis and symbiosis. Science 200I, 292: 1096-1098.

3. Moran NA: Accelerated evolution and Muller's rachet in endosymbiotic bacteria. Proc Natl Acad Sci USA 1996, 93:2873-2878.

4. Ochman H, Moran NA: Genes lost and genes found: evolution of bacterial pathogenesis and symbiosis. Science 200I, 292:1096-1099.

5. Lockhart PJ, Novis P, Milligan BG, Riden J, Rambaut A, Larkum AWD: Heterotachy and Tree Building: A Case Study with Plastids and Eubacteria. Mol Biol Evol 2006, 23:40-45.

6. Andersson SG, Kurland CG: Reductive evolution of resident genomes. Trends Microbiol 1998, 6:263-268.

7. Gil R, Latorre A, Moya A: Bacterial endosymbionts of insects: insights from comparative genomics. Environ Microbiol 2004, 6: I109-1122.

8. Moran NA, Mira A: The process of genome shrinkage in the obligate symbiont Buchnera aphidicola. Genome Biol 200I, 2:RESEARCH0054.

9. Nakabachi A, Yamashita A, Toh $H$, Ishikawa $H$, Dunbar HE, Moran NA, Hattori M: The 160-kilobase genome of the bacterial endosymbiont Carsonella. Science 2006, 3 I 4:267.

10. Dale C, Wang B, Moran N, Ochman H: Loss of DNA recombinational repair enzymes in the initial stages of genome degeneration. Mol Biol Evol 2003, 20: I I 88-1 I 94.

II. Rio RV, Lefevre C, Heddi A, Aksoy S: Comparative genomics of insect-symbiotic bacteria: influence of host environment on microbial genome composition. Appl Environ Microbiol 2003, 69:6825-6832.

12. Toh H, Weiss BL, Perkin SA, Yamashita A, Oshima K, Hattori M, Aksoy S: Massive genome erosion and functional adaptations provide insights into the symbiotic lifestyle of Sodalis glossinidius in the tsetse host. Genome Res 2006, 16:149-156.

13. Carpenter EJ: Marine cyanobacterial symbioses. Biology and environment. Proceedings of the Royal Irish Academy 2002, I02B I:I5-I8.

14. Marin B, Nowack EC, Melkonian M: A plastid in the making: evidence for a second primary endosymbiosis. Protist 2005, 156:425-432.

15. Rai AN, Söderbäck E, Bergman B: Cyanobacterium-plant symbioses. Tansley Review No. 1 I 6. New Phytol 2000, 147:449-48I.

16. Schnepf E, Schlegel I, Hepperle D: Petalomonas sphagnophila (Euglenophyta) and its endocytobiotic cyanobacteria : a unique form of symbiosis. Phycologia 2002, 41:153-157.

17. Kneip C, Lockhart P, Voss C, Maier UG: Nitrogen fixation in eukaryotes--new models for symbiosis. BMC Evol Biol 2007, 7:55.

18. Prechtl J, Kneip C, Lockhart P, Wenderoth K, Maier UG: Intracellular Spheroid Bodies of Rhopalodia gibba Have Nitrogen-Fixing Apparatus of Cyanobacterial Origin. Mol Biol Evol 2004, 21:|477-|48|. 
19. Floener L, Bothe H: Nitrogen fixation in Rhopalodia gibba; a diatom containing blue-greenish inclusions symbiotically. In Endocytobiology; Endosymbiosis and Cell Biology Edited by: Schwemmler W, Schenk HEA. Berlin: Walter de Gruyter \& Co; 1985:54I-552.

20. Masepohl B, Scholisch K, Gorlitz K, Kutzki C, Bohme H: The heterocyst-specific fdxH gene product of the cyanobacterium Anabaena sp. PCC 7120 is important but not essential for nitrogen fixation. Mol Gen Genet 1997, 253:770-776.

21. Huson DH, Bryant D: Application of phylogenetic networks in evolutionary studies. Mol Biol Evol 2006, 23:254-267.

22. Andersson SG, Zomorodipour A, Andersson JO, Sicheritz-Ponten T, Alsmark UC, Podowski RM, Naslund AK, Eriksson AS, Winkler $\mathrm{HH}$, Kurland CG: The genome sequence of Rickettsia prowazekii and the origin of mitochondria. Nature 1998, 396:133-140.

23. Smith KC: Recombinational DNA repair: the ignored repair systems. Bioessays 2004, 26:1322-1326.

24. Andersson JO, Andersson SG: Insights into the evolutionary process of genome degradation. Curr Opin Genet Dev 1999, 9:664-67I.

25. Simonsen R: The diatom system. Ideas on phylogeny. Bacillaria 1979, 2:9-72.

26. Dos Santos PC, Smith AD, Frazzon J, Cash VL, Johnson MK, Dean DR: Iron-sulfur cluster assembly: NifU-directed activation of the nitrogenase Fe protein. J Biol Chem 2004, 279:19705-197II.

27. Silva FJ, Latorre A, Moya A: Genome size reduction through multiple events of gene disintegration in Buchnera APS. Trends Genet 200I, 17:615-618.

28. Dagan T, Blekhman R, Graur D: The "domino theory" of gene death: gradual and mass gene extinction events in three lineages of obligate symbiotic bacterial pathogens. Mol Biol Evol 2006, 23:310-316.

29. Lawrence JG, Hendrix RW, Casjens S: Where are the pseudogenes in bacterial genomes? Trends in Microbiology 200I 9:535-540.

30. Khachane AN, Timmis KN, Martins dSV: Dynamics of reductive genome evolution in mitochondria and obligate intracellular microbes. Mol Biol Evol 2007, 24:449-456.

3I. Lucinski R, Jackowski G: The structure, functions and degradation of pigment-binding proteins of photosystem II. Acta Biochim Pol 2006, 53:693-708.

32. Tamas I, Klasson LM, Sandstrom JP, Andersson SG: Mutualists and parasites: how to paint yourself into a (metabolic) corner. FEBS Lett 200I, 498: I35-139.

33. Sambrook J, Fritsch EF, Maniatis T: Molecular Cloning: A laboratory manual Cold Spring Harbor Laboratory Press; 1998.

34. Sequencher: Gene Codes Corporation. 2006 [http:// www.sequencher.com].

35. Nix DA, Eisen MB: GATA: a graphic alignment tool for comparative sequence analysis. BMC Bioinformatics 2005, 17:9.

36. Guindon S, Gascuel O: A simple, fast, and accurate algorithm to estimate large phylogenies by maximum likelihood. Syst Biol 2003, 52:696-704.

37. Huson DH, Bryant $\mathrm{D}$ : Application of phylogenetic networks in evolutionary studies. Molecular Biology and Evolution 2006, 23:254-267.

38. Akman L, Yamashita A, Watanabe H, Oshima K, Shiba T, Hattori M, Aksoy S: Genome sequence of the endocellular obligate symbiont of tsetse flies, Wigglesworthia glossinidia. Nat Genet 2002, 32:402-407.

39. Shigenobu S, Watanabe H, Hattori M, Sakaki Y, Ishikawa H: Genome sequence of the endocellular bacterial symbiont of aphids Buchnera sp. APS. Nature 2000, 407:8I-86.

40. Stephens RS, Kalman S, Lammel C, Fan J, Marathe R, Aravind L, Mitchell W, Olinger L, Tatusov RL, Zhao Q, Koonin EV, Davis RW Genome sequence of an obligate intracellular pathogen of humans: Chlamydia trachomatis. Science 1998, 282:754-759.

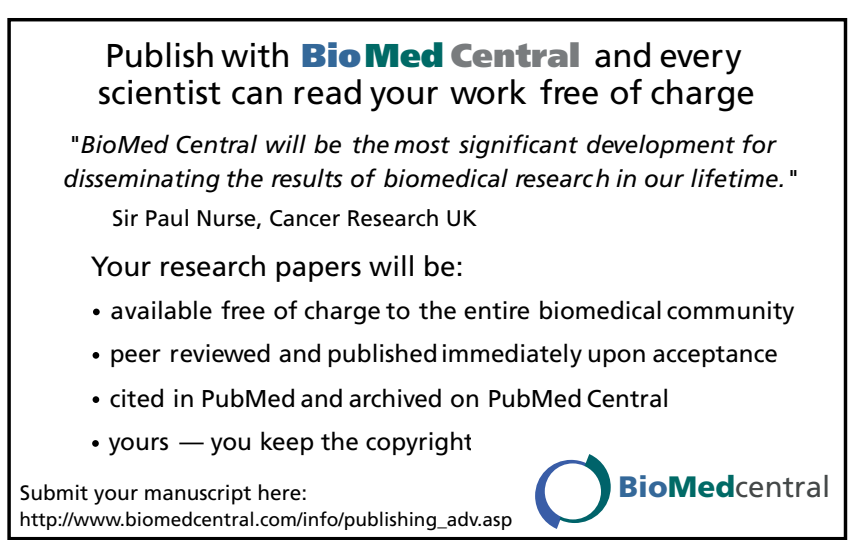

